\title{
Analysis of Normal and Continuous Adventitious Sounds for the Assessment of Asthma
}

\author{
M. Lozano ${ }^{1,4}$, J.A. Fiz ${ }^{2,4,5}$ and R. Jané $e^{3,4,5}$ \\ ${ }^{1}$ Innovation Group at Health Sciences Research Institute of the Germans Trias i Pujol Foundation (IGTP), Badalona, Spain \\ ${ }^{2}$ Pneumology Service at the Germans Trias i Pujol University Hospital, Badalona, Spain \\ ${ }^{3}$ Department of Automatic Control (ESAII), Universitat Politècnica de Catalunya (UPC), Barcelona, Spain \\ ${ }^{4}$ Institute for Bioengineering of Catalonia (IBEC), Barcelona, Spain \\ ${ }^{5}$ Biomedical Research Networking Center in Bioengineering, Biomaterials, and Nanomedicine (CIBER-BBN), Spain
}

\begin{abstract}
Assessment of asthma is a difficult procedure which is based on the correlation of multiple factors. A major component in the diagnosis of asthma is the assessment of BD response, which is performed by traditional spirometry. In this context, the analysis of respiratory sounds (RS) provides relevant and complementary information about the function of the respiratory system. In particular, continuous adventitious sounds (CAS), such as wheezes, contribute to assess the severity of patients with obstructive diseases. On the other hand, the intensity of normal RS is dependent on airflow level and, therefore, it changes depending on the level of obstruction. This study proposes a new approach to RS analysis for the assessment of asthmatic patients, by combining the quantification of CAS and the analysis of the changes in the normal sound intensity-airflow relationship. According to results obtained from three patients with different characteristics, the proposed technique seems more sensitive and promising for the assessment of asthma.
\end{abstract}

Keywords - asthma, bronchodilator response, continuous adventitious sound, respiratory sound intensity, wheezes.

\section{INTRODUCTION}

Asthma is a complex respiratory disorder that results in a variable, recurring, and often reversible airflow obstruction [1]. Physicians have difficulties in diagnosing asthma, since they have to correlate several aspects: the medical history, a thorough physical examination, and pulmonary function test results. In this context, the bronchodilator (BD) response is a standard pulmonary function test used to control and assess the severity of asthma. It is based on spirometric measurements before and after the administration of a BD. Usually, an improvement in forced expiratory volume in one second $\left(\mathrm{FEV}_{1}\right)$ of greater than or equal to $12 \%$ is considered to be significant $[2,3]$. However, some recent studies have demonstrated that using spirometric criteria alone is inadequate for the diagnosis of asthma [4].

Respiratory sounds (RS) are helpful in understanding the function of the respiratory system. They are classified as normal or adventitious sounds. Due to their clinical interest, many technical studies have tried to detect and characterize continuous adventitious sounds (CAS) [5-8], such as wheezes. CAS are characterized by a pitch of over $100 \mathrm{~Hz}$ that lasts more than $100 \mathrm{~ms}$ [9], and they are key indicators for assessing the severity of asthma [1]. On the other hand, some other studies have tried to understand the origin of normal RS and their intensity pattern distribution [10-12]. Although results from all previous studies have contributed to the characterization and understanding of RS, there is lack of clinical use and application of these techniques.

In this study, we propose a new approach to the analysis of RS for the assessment of asthmatic patients, by combining the quantification of CAS and the analysis of the normal sound intensity-airflow relationship. A few previous studies have focused on the evaluation of asthma by RS analysis [13-15]. Nevertheless, some were performed on infants and they were based on manual detection of wheezes and their characterization at a fix airflow level or during forced breathing. On the other hand, in [15] they focused on changes in the spatial distribution of breath sound intensity by analyzing dynamic images. Our technique has two major advantages: the automatic differentiation and quantifying of respiratory cycles either with normal sounds or CAS [16], and the analysis of normal RS intensity as a function of airflow level.

\section{Methods}

\section{A. Signal acquisition}

RS signals were recorded from asthmatic patients in a sitting position at the Pulmonary Function Testing Laboratory, Germans Trias i Pujol University Hospital, Badalona, Spain. Three piezoelectric contact microphones (TSD108, Biopac, Inc.) were attached to the skin using adhesive rings: two of them on the back at $3 \mathrm{~cm}$ below the left/right shoulder blade, and one over the right side of the trachea. Moreover, respiratory airflow signal was recorded using a pneumotachograph (TSD107B, Biopac, Inc.). Each patient was coached to progressively increase the airflow, from shallow breathing to the deepest breaths they could. All 
signals were sampled at 12500 samples/second using a 16bit analogue-to-digital converter. After acquisition, RS signals were band-pass filtered $(70-2000 \mathrm{~Hz})$. We show a case study with three adult asthmatic patients with different baseline spirometric values and BD response (Table 1). For each patient, we have quantified the percentage of respiratory cycles with CAS at baseline and after $\mathrm{BD}$, for both left and right sides. In addition, we have analyzed the relationship between RS intensity and airflow, before and after BD.

Table 1 Characteristics of asthmatic patients

\begin{tabular}{|c|c|c|c|c|c|c|c|c|c|}
\hline \multirow[t]{2}{*}{ ID } & \multicolumn{2}{|c|}{$\begin{array}{l}\text { \# cycles } \\
\text { Pre-BD }\end{array}$} & \multicolumn{2}{|c|}{$\begin{array}{l}\text { \# cycles } \\
\text { Post-BD }\end{array}$} & \multirow[t]{2}{*}{ Age } & \multirow[t]{2}{*}{ Sex } & \multirow{2}{*}{$\begin{array}{c}\mathrm{BMI} \\
\left(\mathrm{Kg} / \mathrm{m}^{2}\right)\end{array}$} & \multirow{2}{*}{$\begin{array}{c}\mathrm{FEV}_{1} \\
(\%)\end{array}$} & \multirow{2}{*}{$\begin{array}{c}\Delta \mathrm{FEV}_{1} \\
(\%)\end{array}$} \\
\hline & Left & Right & Left & Right & & & & & \\
\hline 1 & 46 & 47 & 53 & 53 & 50 & $F$ & 24.44 & 47 & 26 \\
\hline 2 & 68 & 66 & 76 & 83 & 60 & $\mathrm{M}$ & 27.08 & 100 & 1 \\
\hline 3 & 59 & 49 & 53 & 50 & 19 & $\mathrm{M}$ & 19.28 & 59 & 6 \\
\hline
\end{tabular}

\section{B. Segmentation of RS signals}

After signal acquisition, respiratory phases were obtained using the airflow signal as the reference for automatic sound signal segmentation. Since airflow is positive during inspiration and negative during expiration, respiratory phases were marked off by means of a robust zero crossing detector. In order to avoid detection of false endpoints, only cycles in which the airflow reached at least $0.35 \mathrm{~L} / \mathrm{s}$ were considered valid cycles. Moreover, two thresholds of 0.2 and 4 seconds were established for minimum and maximum durations of respiratory phases, respectively. In addition, a threshold of 0.5 seconds was fixed for the maximum time interval between the end of inspiration and the beginning of the corresponding expiration. Two final datasets, pre-BD and post-BD, were obtained for each patient and each side. Each dataset was formed by audio-visual selection of sound signals from the inspiratory cycles, avoiding artifacts such as those from speaking, swallowing, coughing or rubbing. We have focused on inspiratory sounds, which are much louder than expiratory sounds on the back, where we recorded the sound signals.

\section{CAS detection and inspiratory cycle classification}

The first step in the analysis of RS is to differentiate respiratory cycles with normal sounds from those with CAS. For that purpose, we made use of an automatic RS classifier, which we had previously developed based on the analysis and feature extraction from instantaneous frequency (IF) of sound signal from each respiratory cycle [16]. Prior to the IF calculation, sound signals were decomposed into narrow-band components by ensemble empirical mode decomposition (EEMD). The core of the proposed classifier is the fact that IF remains almost constant when a CAS signal is within a respiratory cycle. By using this classifier, we quantified the number of CAS cycles from both pre-BD and post-BD datasets, for each patient and from both left and right sides. Then, we evaluated whether the percentage of CAS cycles had significantly increased or decreased with the administration of the BD.

\section{RS intensity - respiratory airflow graphs}

In addition to evaluate the changes in the percentage of CAS cycles pre/post-BD, we also analyzed the changes in normal sound intensity. Sound intensity was calculated for inspiratory sound segments corresponding to the top $20 \%$ of airflow from each normal sound cycle, from both pre-BD and post-BD datasets. It was defined as the mean power obtained from the power spectral density (PSD) in the frequency band 75-600 Hz. PSD was calculated using Welch's periodogram, with a Hanning window of $80 \mathrm{~ms}$, a $50 \%$ overlap between adjacent segments, and 2048 points for the Fast Fourier Transform. Then, each normal sound cycle was determined by its intensity and the maximum airflow reached. Since each patient was characterized by the relationship between normal sound intensity and airflow on both left and right sides, we analyzed the changes in these graphs in order to evaluate the BD effect.

\section{RESULTS}

In this section we show results from applying the previous techniques to patients shown in Table 1. Firstly, results from respiratory cycle classification and CAS cycle quantification are shown in Fig. 1.

Secondly, we calculated RS intensity from normal sound cycles as a function of the airflow, pre-BD and post-BD, for both left and right sides (Fig. 2).

The patient with severe asthma (ID 1) had 35\% (left) and $30 \%$ (right) of CAS cycles before BD, as shown in Fig. 1. It is in agreement with her low baseline $\mathrm{FEV}_{1}(47 \%)$, which shows that she had a severe bronchial obstruction. After $\mathrm{BD}$, she had not CAS cycles, which is in agreement with her increased $\mathrm{FEV}_{1}\left(\triangle \mathrm{FEV}_{1}=26 \%\right)$. Moreover, normal sound intensity significantly increased after $\mathrm{BD}$, in both sides, as shown in Fig. 2-A.

Contrary to ID1, patient with mild asthma (ID 2) had a normal baseline $\mathrm{FEV}_{1}(100 \%)$, and a low number of CAS cycles $(<1.5 \%)$ before and after BD, as shown in Fig. 1. Moreover, he was a non-responder to $\mathrm{BD}\left(\Delta \mathrm{FEV}_{1}=1 \%\right)$, which was reflected in a very low increase of normal sound intensity, in both sides (Fig. 2-B). 

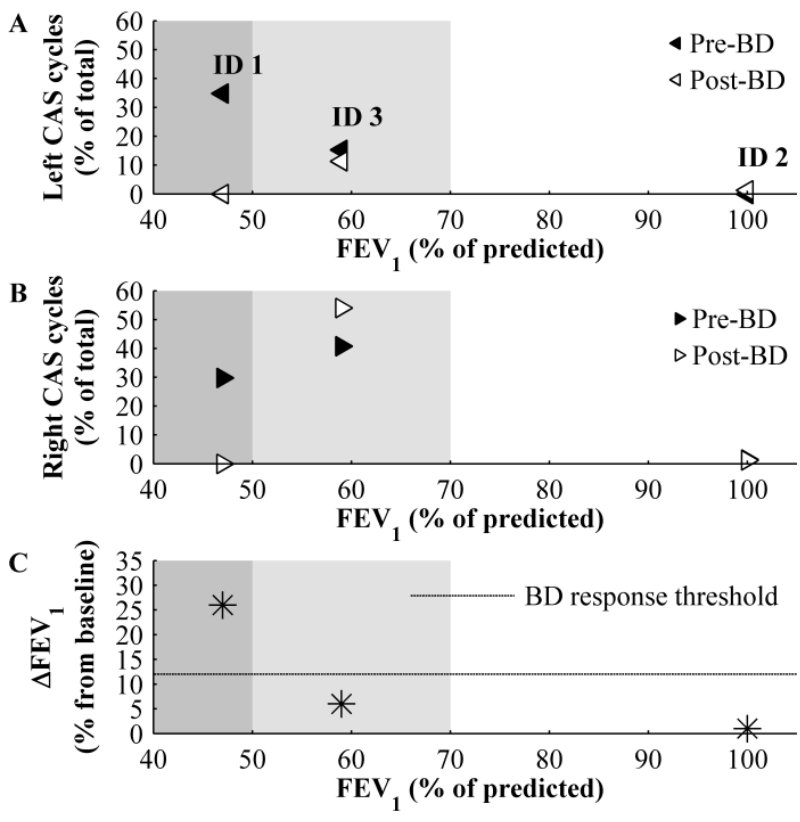

Fig. 1 Percentage of CAS cycles in both left and right sides, at baseline (pre-BD) and after the BD (post-BD) (A, B). Change in $\mathrm{FEV}_{1}$ after BD, as a per cent from baseline, and threshold for a significant $\mathrm{BD}$ response $(12 \%)$

(C). Three degrees of severity were defined based on baseline $\mathrm{FEV}_{1}$ : mild $(x \geq 70 \%)$, moderate $(50 \% \leq x<70 \%)$, and severe $(x<50 \%)$.

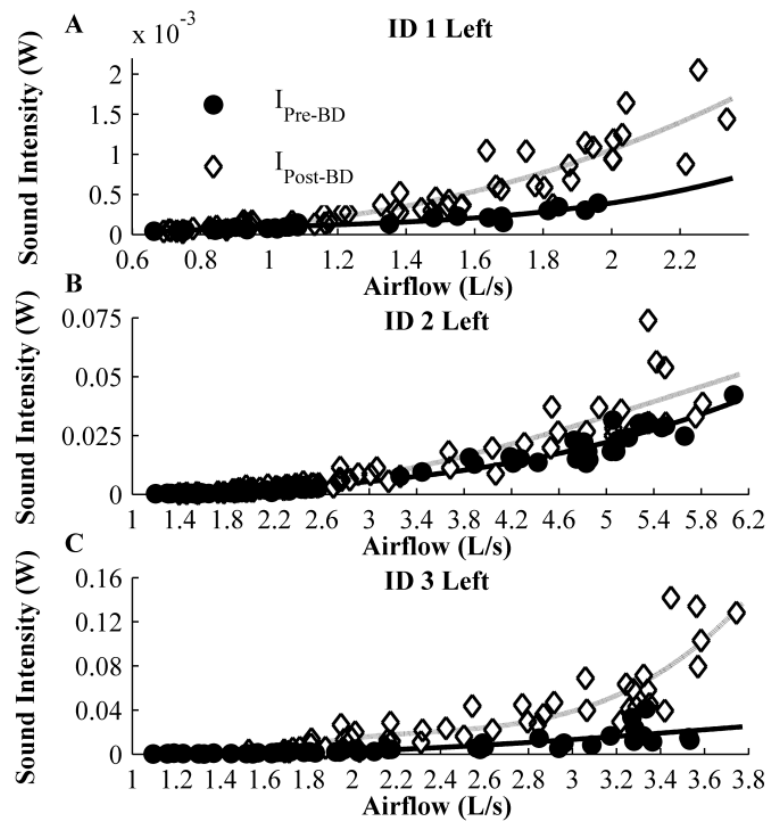

In addition to the previous extreme cases, we analyzed an intermediate case with moderate asthma (ID 3). He was a slight responder to $\mathrm{BD}$, since he had a $\triangle \mathrm{FEV}_{1}$ of $6 \%$. However, he had a baseline $\mathrm{FEV}_{1}$ of $59 \%$, which is in the range of moderate-severe asthma. It agrees with his $15 \%$ (left) and $41 \%$ (right) of CAS cycles at baseline. After BD, the percentages of CAS cycles were $11 \%$ (left) and $54 \%$ (right), which were maintained high values. Although his BD response was not significant, Fig. 2-C shows that he had a relevant increase in normal sound intensity that was clearer at high airflows.

\section{Discussion}

According to the aforementioned results, the baseline $\mathrm{FEV}_{1}$ is related to the number of CAS cycles. Those patients with a low baseline $\mathrm{FEV}_{1}$ have high probability of having wheezes, and vice versa.

On the other hand, the changes in the number of CAS and normal sound intensity are more related to the BD response. A positive BD response indicates that the bronchial obstruction has significantly decreased and, therefore, there are less CAS cycles and higher normal sound intensity. On the contrary, non-responders do not have many changes in their bronchial tree, which implies having few changes in the number of CAS cycles and low increase of their normal sound intensity.

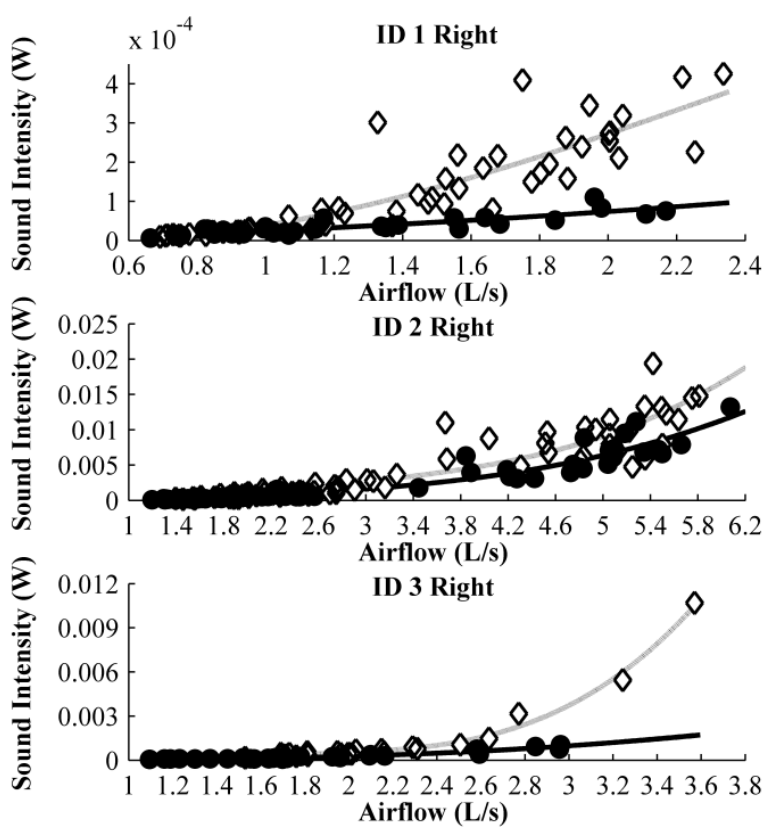

Fig 2. Comparison between normal sound intensity and airflow before and after the administration of a BD. For each patient, results from both left and right sides are shown. Solid and dotted lines show the $3^{\text {rd }}$ order polynomial regression models that fit pre-BD and post-BD intensities as functions of airflow, respectively. 
Moreover, we have shown that for a patient with a nonsignificant BD response (below 12\%), the analysis of normal sound intensity is a more sensitive technique, since significant increases can be detected after BD. Furthermore, these increases are more relevant at high airflow levels.

With respect to the proposed technique, it has some advantages: the exploration and evaluation of the pulmonary function at different airflow levels, which are reached during the progressive respiratory maneuver, and the use of two recording channels that allow us to obtain information about the laterality of normal RS and CAS.

\section{v. Conclusions}

In this study we have shown that the combined analysis of normal RS and CAS provides a promising approach to characterize asthmatic patients. It is a simple and noninvasive technique, which seems more sensitive to changes in the pulmonary function. Therefore, it could be a complementary tool in the diagnostic procedure for asthma. However, this work is a case-study with three patients and therefore a higher number of varied cases are required in order to draw final conclusions.

\section{AcKNOWLEDGMENT}

This work was made possible thanks to a collaboration agreement between IBEC and IGTP, and it was supported in part by the Spanish Ministry of Economy and Competitiveness under grant TEC2010-21703-C03-01. All authors would like to thank Rosa Gómez, from the pulmonary function testing laboratory at Germans Trias i Pujol University Hospital, for its collaboration in the patient recruitment.

\section{REFERENCES}

1. National Heart, Lung, and Blood Institute (2007) National asthma education and prevention program. Expert panel report 3: Guidelines for the diagnosis and management of asthma. http://www.ncbi.nlm.nih.gov/books/NBK7232/

2. Reddel HK, Taylor DR, Bateman ED et al. (2009) An official American Thoracic Society/European Respiratory Society statement: asthma control and exacerbations: standardizing endpoints for clinical asthma trials and clinical practice. Am J Respir Crit Care Med 180:59-99

3. Pellegrino R, Viegi G, Brusasco V et al. (2005) Interpretative strategies for lung function tests. Eur Respir J 26:948-968

4. Gjevre JA, Hurst TS, Taylor-Gjevre RM, Cockcroft DW (2006) The American Thoracic Society's spirometric criteria alone is inadequate in asthma diagnosis. Can Respir J 13:433-437

5. Bahoura M (2009) Pattern recognition methods applied to respiratory sounds classification into normal and wheeze classes. Comput Biol Med 39:824-843
6. Homs-Corbera A, Fiz JA, Morera J, Jané R (2004) Time-frequency detection and analysis of wheezes during forced exhalation. IEEE Trans Biomed Eng 51:182-186

7. Taplidou SA, Hadjileontiadis LJ (2010) Analysis of wheezes using wavelet higher order spectral features. IEEE Trans Biomed Eng 57:1596-1610

8. Xie S, Jin F, Krishnan S, Sattar F (2012) Signal feature extraction by multi-scale PCA and its application to respiratory sound classification. Med Biol Eng Comput 50:759-768

9. Sovijärvi ARA, Dalmasso F, Vanderschoot J et al. (2000) Definitions of terms for applications of respiratory sounds. Eur Respir Rev 10:597-610

10. Murphy R (2007) Computerized multichannel lung sound analysis. Development of acoustic instruments for diagnosis and management of medical conditions. IEEE Eng Med Biol Mag 26:16-19

11. Pasterkamp H, Consunji-Araneta R, Oh Y, Holbrow J (1997) Chest surface mapping of lung sounds during methacholine challenge. Pediatr Pulmonol 23:21-30

12. Torres-Jimenez A, Charleston-Villalobos S, Gonzalez-Camarena R, Chi-Lem G, Aljama-Corrales T (2008) Asymmetry in lung sound intensities detected by respiratory acoustic thoracic imaging (RATHI) and clinical pulmonary auscultation. Conf Proc IEEE Eng Med Biol Soc 2008:4797-4800

13. Sánchez I, Vizcaya C, García D, Campos E (2005) Response to bronchodilator in infants with bronchiolitis can be predicted from wheeze characteristics. Respirology 10:603-608

14. Mazic I, Sovilj S, Magjarevic R (2003) Analysis of respiratory sounds in asthmatic infants. Meas Sci Rev 3:9-12

15. Guntupalli KK, Reddy RM, Loutfi RH et al. (2008) Evaluation of obstructive lung disease with vibration response imaging. J Asthma 45:923-930

16. Lozano M, Fiz JA, Jané R (2013) Estimation of instantaneous frequency from empirical mode decomposition on respiratory sounds analysis. In: Proceedings of the 35th International Conference of the IEEE Engineering in Medicine and Biology Society, Osaka (in press)

Corresponding author:

Author: Manuel Lozano

Institute: Institut for Bioengineering of Catalonia

Street: Baldiri Reixac, 4, Torre I, 9th floor, 08028

City: Barcelona

Country: Spain

Email: mlozano@ibecbarcelona.eu 